\title{
APOE $\epsilon 4$ allele, age, and duration of unconsciousness were associated with unfavourable outcomes in traumatic brain injury
}

Friedman G, Froom P, Sazbon L, et al. Apolipoprotein E-\&4 genotype predicts a poor outcome in survivors of traumatic brain injury. Neurology 1999 Jan 15;52:244-8.

\section{Question}

In patients who survive traumatic brain injury (TBI), what is the relation between the apolipoprotein $\mathrm{E}$ (APOE) $\varepsilon 4$ allele and outcome?

\section{Design}

Cohort analytic study with $6-8$ months of follow up.

\section{Setting}

A hospital department for brain injury and rehabilitation and an outpatient rehabilitation clinic in Israel.

\section{Participants}

69 patients (mean age $36 \mathrm{y}, 75 \%$ men) with blunt trauma. Patients with penetrating injuries or anoxic brain damage were excluded.

\section{Assessment of risk factors}

APOE genotype, Glasgow Coma Scale score, duration of unconsciousness, admission to an intensive care unit (ICU) in hospital, age, and duration of education.

\section{Main outcome measures}

Independence in activities of daily living (ADL), cognitive and behavioural abnormalities, dysarthria, dysphasia, epilepsy, and overall function. A good outcome was defined as no dysarthria, behavioural abnormalities, or dysphasia; no severe cognitive abnormalities; and the ability to live independently. Outcome assessors were blinded to APOE status.

\section{Main results}

Patients who were unconscious for $>7$ days were more likely to have an APOE $\varepsilon 4$ allele $(78 \% v 38 \%, \mathrm{p}=0.001)$. Fewer patients with an APOE $\varepsilon 4$ allele had a good outcome than those without such an allele ( $4 \% v 31 \%, \mathrm{p}=0.006$, odds ratio [OR] 0.1, 95\% CI 0.0 to 0.7 ). More patients with an APOE $\varepsilon 4$ allele had dysarthria $(63 \%$ v 33\%, $\mathrm{p}=0.02$, OR 3.4, CI 1.1 to 10.7$)$. No statistically significant associations existed between the presence of an APOE $\varepsilon 4$ allele and independence in ADL, behavioural abnormalities, severe cognitive abnormalities, dysarthria, or epilepsy. In multivariate analysis, the independent risk factors for an unfavourable outcome were the presence of an APOE $\varepsilon 4$ allele $(\mathrm{p}=0.02)$, increasing age $(\mathrm{p}=0.01)$, and being unconscious for $>7$ days $(\mathrm{p}=0.02)$ (table).

\section{Conclusions}

In patients who survived traumatic brain injury, those having an apolipoprotein $\varepsilon 4$ allele were more likely to be unconscious for $>7$ days. At 6 months, independent risk factors for poor outcome were presence of the APOE $\varepsilon 4$ allele, older age, and unconsciousness for $>7$ days.

Risk factors for an unfavourable outcome in patients who have survived traumatic brain injury

\begin{tabular}{ll}
\hline Risk factors & Adjusted odds ratio $(95 \% \text { CI })^{*}$ \\
\hline APOE $\varepsilon 4$ allele present & $13.9(1.5$ to 134.0$)$ \\
Age & $1.1(1.0$ to 1.2$)$ per year \\
Unconscious for $>7$ days & $7.6(1.4$ to 40.0$)$ \\
\hline
\end{tabular}

*Adjusted for Glasgow Coma Scale score, duration of unconsciousness, age, and education. Source of funding: HJ Leir

For correspondence: $D r$ Z Groswasser, Department of Brain Injury Rehabilitation, Loewenstein Rehabilitation Hospital, 278 Achuza Street, Ra'anana 43100, Israel. Fax +97297709998

\section{Commentary}

Psychiatrists working in rehabilitation settings and in general hospitals frequently have to assess the prognosis of patients with TBI. This task is a challenging one because long term outcome depends on an interaction of susceptibility to injury, severity of insult, and capacity to repair and regenerate. To date, the prediction of long term outcome has relied largely on measures of short term outcome, such as duration of unconsciousness or post-traumatic amnesia, or coma score. ${ }^{1}$ Risk factors that are not themselves aspects of outcome offer hope of more accurate and earlier prediction. Such factors are likely to relate to susceptibility to injury or to repair.

Investigation of the biochemical and genetic basis of neuronal growth and repair has led to the identification of the $\varepsilon 4$ allele of APOE as one potential factor. Its clinical relevance has been confirmed in the degenerative brain disorder Alzheimer's disease, for which APOE $\varepsilon 4$ is a risk factor, at least in whites. ${ }^{2}$ This study by Friedman et al extends previous work on TBI and APOE $\varepsilon 4$, and provides further support for the role of this allele in the determination of prognosis.

However, there are 2 clear caveats. Firstly, the influence of race on the association between this allele and outcome from TBI is not yet known. In Alzheimer's disease, the association seems much clearer among whites than other racial groups. Secondly, the magnitude of the association awaits further clarification. The confidence intervals around the point estimate of effect are very wide, such that it is quite possible that the odds ratio for the allele is much higher or much lower than that for $>7$ days of unconsciousness. It is therefore too early to say whether this study marks a step forward in the prognostic assessment of TBI.

At the very least, however, this study should stimulate further research and encourage clinicians with relevant interests to maintain a watchful and critical eye on the growing literature about APOE $\varepsilon 4$.

Jonathan Price, MA, MRCPsych University of Oxford Oxford, UK

1 Asikainen I, Kaste M, Sarna S. Brain Inj 1998;12:95-107.

2 Tang MX, Stern Y, Marder K, et al. JAMA 1998; 279:751-5. 